\title{
Thermal Properties of Doped Azopolyester and its Application
}

\author{
K. Gopalakrishnan1*, M. Prem Jeya Kumar', J. Sundeep Aanand ${ }^{3}$ and R. Udayakumar ${ }^{4}$ \\ 'Professor of Electronics Communication Engineering, Bharath University, Chennai-600073; \\ gopalkrishnan.ece@bharathuniv.ac.in \\ 2Professor of Automobile Engineering, Bharath University, Chennai; premjeyakumar.coe@bharathuniv.ac.in \\ ${ }^{3}$ Professor of Computer Science Engineering, Bharath University, Chennai; sundeepssn@hotmail.com \\ ${ }^{4}$ Associate Professor of Information Technology, Bharath University, Chennai; rsukumar2007@hotmail.com
}

\begin{abstract}
The thermal properties of doped Azopolyester were obtained by solution casting technique. Many theories have been suggested by various thermal stability mechanisms in organic solids. Since the polymers of the present study behave like semiconductors, these theories may be extended to explain thermal stability. In this section, the thermal stability theories proposed by O'Dwyer, TGA analysis, and measurements were discussed. The thermal stability properties reveal that the conductivity is appreciable in the temperature ranging from $30^{\circ} \mathrm{C}$ to $70^{\circ} \mathrm{C}(303$ to $343 \mathrm{~K})$ and when it is more than $70^{\circ} \mathrm{C}(343 \mathrm{~K})$ the conductivity is drastically reduced and polymeric blends undergo a gradual weight loss in the temperature ranging from $400-800^{\circ} \mathrm{C}$ due to attributed loss of volatile solvents trapped in the polymer. These studies are done with the help of O’Dwyer basic theory of thermal stability.
\end{abstract}

Keywords: TGA, Thermal Properties, Azopolyester, Conductivity, TGA analysis.

\section{Introduction}

The phenomenon of thermal stability is an interesting behaviour of polymer thin films. This property is very useful to study the behavior of electrical, optical and structural properties of the doped Azopolyester thin films and their applications [1-7]. In all these applications the thermal stability of the doped Azopolyester film is an important factor. Essential making the study of thermal property of the doped Azopolyester films [8-14]. These investigations play an important role in the systematic development of devices [15]. The different theories proposed so far, observed to establish whether the thermal stability results from a local event or whether it is the consequence of a phenomenon propagating with an increase of intensity. Experiments have been performed on samples subjected to temperature range from $300 \mathrm{~K}-800 \mathrm{~K}$, pulses of electrical voltage [16-17] or samples biased in the pre thermal stability region [18]. Extensive research has been done on the thermal properties of doped Azopolyesters [19-22]. The criterion that determines thermal stability is a form of self biasing due to temperature.

This chapter deals with the thermal properties of doped Azopolyester prepared by solution casting technique. Many theories have been suggested by various thermal stability mechanisms in organic solids. Since the polymers of the present study behave like semiconductors, these theories may be extended to explain thermal stability. In this section, the thermal stability theories proposed by O'Dwyer, TGA analysis, and measurements were discussed.

\section{Basic Theory of O'Dwyer Thermal Stability}

O' Dwyer theory incorporates a condition of current continuity during avalanche (thermal) process which causes the field to be heterogeneous, being considerably larger at the

*Corresponding author:

K. Gopalakrishnan (gopalkrishnan.ece@bharathuniv.ac.in) 
cathode than elsewhere. The thermal stability mechanism is associated with impact ionization [23]. The instability is due to the positive feedback between the holes, which are trapped by impact ionization and the electrons which are injected from the cathode [24]. The injection is enhanced by trapped holes owing to the increase in field at the cathode. This result is an increase in impact ionization and hole trapping [25]. When this rise cannot be balanced by whole drift, a break down occurs. Thermal studies of conducting polymers are particularly important when one considers the use of elevated temperatures to process these polymers and their blends into technologically useful in various optical and electronic applications. The thermal stability of a polymer has a strong influence on the characteristic melting and crystallization temperatures [26].

\section{Thermo Gravimetric Analysis}

Thermal analyses of doped Azopolyester are studied by Thermo Gravimetric Analysis (TGA). It is used to measure changes in the molecular weight, weight loss of a material as a function of temperature in the scanning mode or as a function of time in the isothermal mode. Thermo gravimetric technique is used to study the thermal stability as well as decomposition of materials under a variety of conditions to examine the kinetics of physiochemical processes occur in the material [27]. The weight change properties of a material strongly depends on the experimental conditions applied [28]. Factors such as sample mass, volume and physical properties, the shape and nature of sample holder, the nature and pressure of the air in the sample holder and the scanning rate all have important factors that influence the properties of the recorded TGA curve [29].

Thermo gravimetric analysis is commonly used to determine polymer withstand (or) degradation temperatures, residual solvent levels, absorbed moisture content, and the amount of inorganic molecule in polymer compositions.

Figure 1 shows the schematic diagram of a thermo gravimetric analyzer consists of heater unit, thermo couple unit to measure sample temperature, pressure gauge to measure sample weight and entire arrangement in a closed envelope filled with inert gas $\left(\mathrm{N}_{2}\right)$ atmosphere. The purpose of inert gas is to prevent oxidation or other chemical reactions. A PC is connected with this unit to take TGA output. The pressure gauge senses the molecular weight of a sample, the pressure variations occurred is converted into corresponding electric signal, it is further converted into digital variations by means of $\mathrm{A} / \mathrm{D}$ convertor, with the

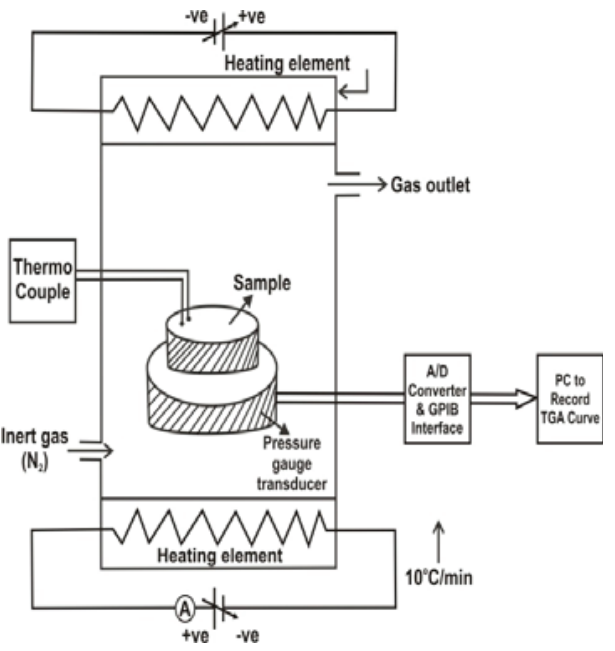

Figure 1. Schematic diagram of a thermo gravimetric analyzer.

help of USB or GPIB interface the output data has been plotted. The present studies are carried out with the help of TGA (Model V3.2) BTA instrument in an inert gas $\left(\mathrm{N}_{2}\right)$ at a heating rate of $10^{\circ} \mathrm{c} / \mathrm{min}$.

\section{Measurement Techniques}

The thermal stability of doped Azopolyester with three different doping of PANI $\left(\right.$ Poly $_{10}$, Poly $_{30}$, and Poly ${ }_{50}$ ) has been studied with the help of pellets under isothermal conditions by using four in line DC electrical conductivity measuring instrument. This study was carried out at $30,40,50,60,70$, 80 , and $120^{\circ} \mathrm{C}$ in an oven. The electrical conductivity measurements were done at an interval of 15 minutes in the accelerating ageing experiment to study the thermal properties of pellets for temperature range from $30^{\circ} \mathrm{C}$ to $800^{\circ} \mathrm{C}$.

\section{Results and Discussion}

The thermal stability behavior of the doped Azopolyester with three weight ratios of 10\%, 30\%, 50\% doping of PANI$\mathrm{HCl}$ blends is shown in the Figure 2.

Thermo gravimetric analysis curves of all the three different doped Azopolyester shows a small weight loss around $100-200^{\circ} \mathrm{C}$. This is due to the loss of water content present in the polymer. Results of thermal stability of the polymeric blends are summarized in table 1.1. It is observed from figure 1.2 that the thermal stability of the 50\% PANI doped Azopolyester is higher value than compared to $10 \%$ and 30\%. The PANI doped Azopolyester with 10\% 


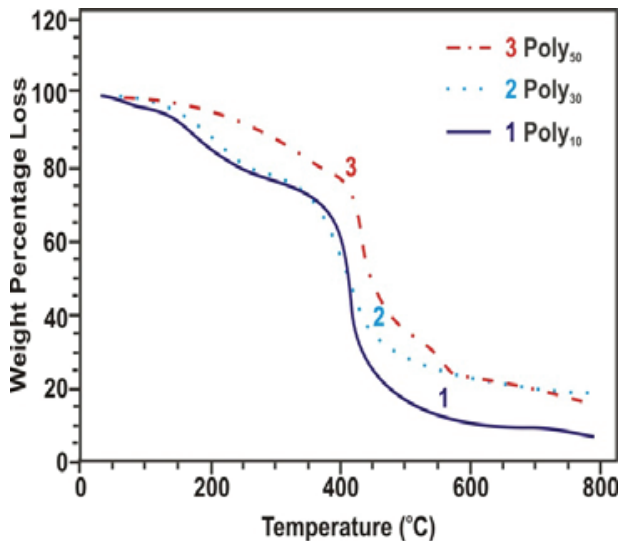

Figure 2. TGA curves of three different doped Azopolyester blends.

weight loss occurred from the blends investigation starts temperature at $190^{\circ} \mathrm{C}, 200^{\circ} \mathrm{C}$ and $290^{\circ} \mathrm{C}$ for poly ${ }_{10}$, poly ${ }_{30}$ and poly ${ }_{50}$ respectively. Similarly $20 \%$ weight loss occurred temperature at $255^{\circ} \mathrm{C}, 290^{\circ} \mathrm{C}$ and $390^{\circ} \mathrm{C}$ for poly ${ }_{10}$, poly ${ }_{30}$ and poly ${ }_{50}$ respectively. Finally $50 \%$ weight loss occurred temperature at $410^{\circ} \mathrm{C}, 420^{\circ} \mathrm{C}$ and $450^{\circ} \mathrm{C}$ for poly ${ }_{10}$, poly ${ }_{30}$ and poly ${ }_{50}$ respectively. The lower thermal stability of the blends is due to rapid dehydration and loss of water molecule of the blends that leads evaporation of dopant $\mathrm{HCl}$ in doped Azopolyester. The weight loss percentage at $450^{\circ} \mathrm{C}$ shown in table 1.1 reveal that the percentage of weight loss decreases when doping percentage of PANI increases in Azopolyester due to attributed loss of volatile solvents trapped in the polymer.

The isothermal stability testing for the three different doped Azopolyester $\left(\right.$ poly $_{10}$, poly $y_{30}$ and poly ${ }_{50}$ ) in terms of direct current, conductivity is carried out at various temperatures ranging from 303 to $393 \mathrm{~K}$ in air atmosphere using oven. The DC conductivity measured with respect to time of accelerated ageing is presented in the Figures 3, 4 and 1.5.

It is observed from the Figures 2, 4 and 5 that the doped Azopolyester conductivity increases when temperature

Table 1. Thermo gravimetric analysis of different doped Azopolyester blends

\begin{tabular}{ccccc}
\hline sample & \multicolumn{2}{c}{ Decomposi. Temperature ${ }^{\circ} \mathrm{C}$} & loss at $450^{\circ} \mathrm{C}$ \\
\cline { 2 - 4 } & $\mathbf{1 0 \%}$ & $\mathbf{2 0} \%$ & $\mathbf{5 0 \%}$ & \\
& Weight & Weight & Weight & \\
& Loss at & Loss at & Loss at & \\
\hline $10 \%$ & $190^{\circ} \mathrm{C}$ & $255^{\circ} \mathrm{C}$ & $410^{\circ} \mathrm{C}$ & $80 \%$ \\
$30 \%$ & $200^{\circ} \mathrm{C}$ & $290{ }^{\circ} \mathrm{C}$ & $420^{\circ} \mathrm{C}$ & $65 \%$ \\
$50 \%$ & $290^{\circ} \mathrm{C}$ & $390{ }^{\circ} \mathrm{C}$ & $450^{\circ} \mathrm{C}$ & $36 \%$ \\
\hline
\end{tabular}

increases from $30-70^{\circ} \mathrm{C}$. Further, when the temperature is more than $70^{\circ} \mathrm{C}(343 \mathrm{~K})$ the conductivity is drastically reduced, due to evaporation of dopant $\mathrm{HCl}$ ion doped Azopolyester.

\section{Conclusion}

The thermal stability properties reveal that the conductivity is appreciable in the temperature ranging from $30^{\circ} \mathrm{C}$

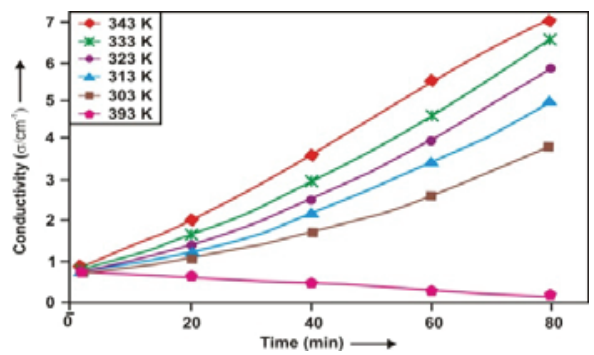

Figure 3. Thermal stability in terms of DC conductivity retention under isothermal conditions at various temperatures for $10 \%$ doped Azopolyester $\left(\right.$ Poly $\left._{10}\right)$.

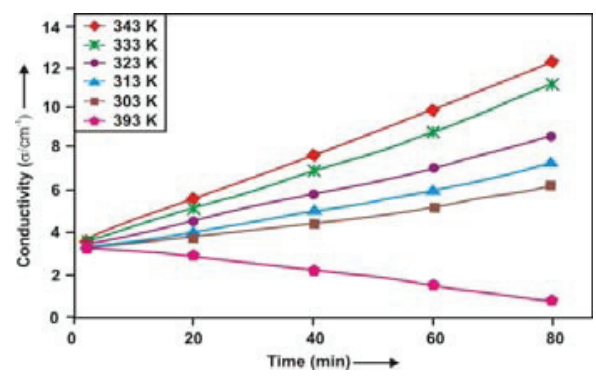

Figure 4. Thermal stability in terms of DC conductivity retention under isothermal conditions at various temperatures for $30 \%$ doped Azopolyester $\left(\right.$ Poly $_{30}$ ).

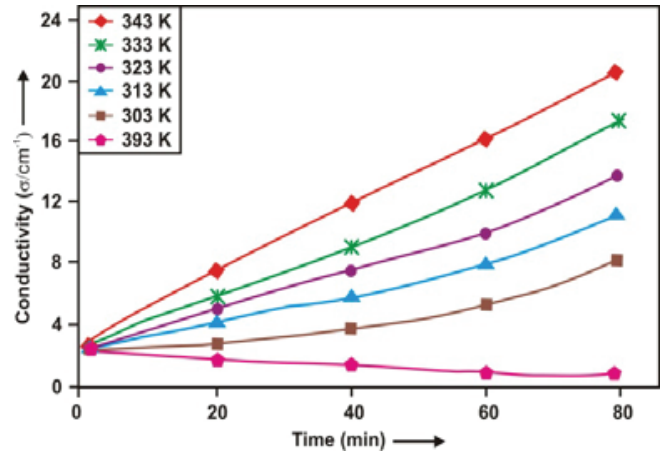

Figure 5. Thermal stability in terms of DC conductivity retention under isothermal conditions at various temperatures for 50\% doped Azopolyester $\left(\right.$ Poly $\left._{50}\right)$. 
to $70^{\circ} \mathrm{C}$ (303 to $343 \mathrm{~K}$ ) and when it is more than $70^{\circ} \mathrm{C}$ $(343 \mathrm{~K})$ the conductivity is drastically reduced and polymeric blends undergo a gradual weight loss in the temperature ranging from $400-800^{\circ} \mathrm{C}$ due to attributed loss of volatile solvents trapped in the polymer.

\section{References}

1. Mori T, Namba $T$ et al. (1994). Electrical breakdown of ethylene-acrylic acid copolymer and blend polymer films, IEEE International Symposium on Conference Record of the 1994, Pittsburgh, USA, 217-220.

2. Klein N (1978). Electrical breakdown mechanisms in thin insulator, Thin Solid Films, Elsevier, vol 50, 223-232.

3. Weaver C, McLeod J E S et al. (1965). Thermal properties of conjugated polymers, Journal of Physics D: Applied Physics, vol 16, 11-16.

4. Zelm M, and Physik Z (1968). Electron avalanches in pellets, Journal of Applied Physics, vol 46, 2946-2987.

5. Copper R, Elliot C T et al. (1966). A photographic study of electrical breakdown of polymer, Journal of Applied Physics, vol 17(17), 169-195.

6. Klein N (1971). Switching and breakdown in films, Thin Solid Films, vol 7(3-4),149-177.

7. Sawa G (1979). Conditions for complete self healing breakdown in glow discharge polymerized styrene films, Thin Solid Films, vol 59(2), 131-141.

8. Sapieha S, Kriyszewski M et al. (1972). International Microsymposium on polarization and conduction in insulating polymers, Bratislava, vol 36, 119-124.

9. Kriyszewski M, Jablonski W et al. (1972). International Microsymposium on polarization and conduction in insulating polymers, Bratislava, vol 41(67), 119-125.

10. Ya V, Airazov et al. (1971). Electrical breakdown and plasma polymerized styrene thin films, Souvenir Physics Technology, vol 16, 1782.

11. Segui Y, Ai Bui et al. (1974). Charge transport in thin polymer films, Thin Solid Films, vol 22(1), 47-140.

12. Nagao M, Sawa G et al. (1977). Pre breakdown currents due to filamentary thermal breakdown in polyimide films, IEEE Transactions on Electrical and Electronic Engineering, 97A, 279-355.

13. Ieda M (1980). Carrier injection, space charge and electrical breakdown in insulating polymers, IEEE Transactions on Electrical Insulation, vol E1-15(3), 206-224.
14. Muramoto Y, Nagao M et al (1995). Self healing breakdown of polyimide thin films in the cryogenic temperature region, Cryogenics, vol 35(11), 791-793.

15. Coppards R W, Bowman J et al. (1990). The effect of aluminium inclusions on the dielectric breakdown of polyethylene, Journal of Physics D: Applied Physics, vol 23, 1554.

16. Paul P, Budenstenin et al. (1969). Origin and evolution of sculptured thin films, Vacuum Science Technology, vol 6, 235-289.

17. Hossain M M (1993). Effect of humidity on the breakdown strength and diffusion characteristics of polymer film, Bulletin of Materials Science, vol 16(6), 699-707.

18. Forlani F, and Minnaja N J (1969). Electrical breakdown in thin dielectric films, Journal of Vacuum Science and Technology, vol 6(4), 518-526.

19. Goldstein R M, and Leonhard F W (1967). Dielectric breakdown study of thin LaZo3 films, Proceedings of $17^{\text {th }}$ electronic components, Washington DC.

20. Balasubramanian T, Narayandass S K et al. (1997). Optical and transport properties of alloy thin films, Indian Journal of Engineering and Materials Sciences, vol 17(35), 149-153.

21. Amarjit Singh (1983). Dielectric breakdown study of thin LaZo3 films, Thin Solid Films, Elsevier, vol 105(88), 163.

22. Dharmadhikari V S, and Goswami A (1982). Dielectric properties of electron beam evaporated NdZo3 thin films, Thin Solid Films, vol 87(13), 117-123.

23. Balasubramanian T, Naryandass S K et al. (1996). Space charge limited conduction in polyaniline films, Bulletin of Indian Vacuum Society, vol 27(16), 23-32.

24. Chambell C K (1970). Some dielectric properties of electron beam evaporated yttrium oxide thin films, Thin Solid Films, vol 6(3), 197-202.

25. Wiktorczyk T, and Wesolowska C (1980). Dielectric properties of yttrium oxide films deposited by electron beam evaporation, Thin Solid Films, vol 71(20), 15-27.

26. Mahalingam T, Radhakrishnan $\mathrm{M}$ et al. (1980). Dielectric behaviour of lanthanum oxide thin film capacitors, Thin Solid Films, vol 74(3), 27-34.

27. Goswami A, and Varma R R (1975). Dielectric behaviour of oxide films, Thin Solid Films, vol 28(2), 157-165.

28. Koleshko V M, and Babushkina N V (1979). Thin films of rare earth metal silicates, Thin Solid Films, vol 62(3), 337-339.

29. Forlani F, and Minnaja M (1964). Physics of thin film ferroelectric oxides, IOP Science: Reports on Progress in Physics, vol 34(1), 311-316. 